\title{
El cambio de conceptos y teorías en el conocimiento científico y ordinario*
}

\author{
Francisco Javier Fuentes Farías \\ (iD $h t t p: / / o r c i d . o r g / 0000-0001-9483-816 X$
}

Universidad Michoacana de San Nicolás de Hidalgo, México

DOI: http://dx.doi.org/10.17081/eduhum.19.33.2643

Recibido: Septiembre 30 de 2016

Aceptado: 27 de febrero de 2017

\section{Change of concepts and theories in scientific and ordinary knowledge}

\author{
Palabras clave: \\ Cognición social, \\ Epistemología genética, \\ Constructivismo, Enactivismo, \\ Fenomenología.
}

Key words:

Social cognition,

genetic epistemology,

constructivism,

power of implementation or enac-

tivism,

phenomenology.

\begin{abstract}
Resumen
Piaget demostró que el desarrollo cognitivo continúa en la vida adulta, y que hay cierta analogía entre el conocimiento ordinario y el científico. Pero, ¿qué tipo de recursos cognitivos son fundamentales en la transmisión del conocimiento científico? Un problema central se refiere al mecanismo de cambio conceptual y de teorías, mismo que abordamos brevemente para mostrar aspectos relevantes de un cambio de paradigma en el conocimiento científico desde un punto de vista cartesiano, positivista, reduccionista y fisicalista, a uno comprensivo e interpretativo, complejo, holista y constructivista. Todo cambio conceptual y de teorías implica heurísticas nuevas para descubrir nuevos aspectos de la realidad, lo cual podría ser un polo de atención en la enseñanza del conocimiento.
\end{abstract}

\begin{abstract}
Piaget demonstrated that cognitive development still continues in adult life, and that there is certain analogy between ordinary and scientific knowledge. But, what type of cognitive resources is fundamental for scientific knowledge transmission? A central problem refers to the mechanism of change of ideological concepts and theories, the same one we tackled briefly in order to show relevant aspects about a change of paradigms in scientific knowledge from a cartesian, positivist, reductionist and physical point of view up to a comprehensive, interpretative, complex, holistic and constructivist one. Every conceptual and theoretical change implies a new heuristic for the search of new aspects of reality, which could be the center of attention for the teaching of any kind of knowledge.
\end{abstract}

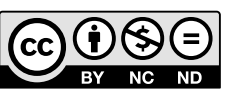

Referencia de este artículo (APA): Fuentes, F. (2017). El cambio de conceptos y teorías en el conocimiento científico y ordinario. En Revista Educación y Humanismo, 19(33), 253-270. http://dx.doi.org/10.17081/eduhum.19.33.2643

\footnotetext{
* Proyecto de investigación: El problema de la socioespacialidad urbano arquitectónica. Institución que soporta: Consejo Nacional de Ciencia y Tecnología (CONACyT).

1. Doctor en Arquitectura Sistema Nacional de Investigadores Nivel I. Profesor de la Facultad de Arquitectura Universidad Michoacana de San Nicolás de Hidalgo. fuentes88@hotmail.com
} 


\section{Introducción}

Durante el siglo anterior hubo diversos movimientos que estudiaron la manera de entender el conocimiento y su organización dentro de los esquemas de la investigación académica y disciplinar. Esto quedó plasmado en diferentes trabajos, como es el caso de la obra de Kuhn, Bertalanffy, Feyerabend, Bachelard, Piaget, Wallerstein, Morin y otros. Un aspecto central de estas investigaciones fue su crítica al modelo del empirismo científico y a las fronteras de demarcación entre conocimiento científico y otros conocimientos, así como la importancia que dieron a la fenomenología, la hermenéutica, la ontología y la semiótica en la comprensión y el conocimiento de las experiencias subjetivas, mentales o psicológicas de las personas.

Al mismo tiempo quedó claro el papel de la filosofía en las teorizaciones sobre el conocimiento. Por ejemplo, con Kuhn, Piaget, Morin o Adorno (De la Garza, 2012a, p.230; Carey, Zaitchik \& Bascandziev, 2015, pp.6, 7; García, 2006, pp.20, 32, 35), cuyas contribuciones orientaron nuevos puntos de vista acerca de la influencia del lenguaje, la sociedad y el poder político-económico en el conocimiento. Con Wittgenstein, por otra parte, se abrió una nueva comprensión y concepción del lenguaje $\mathrm{y}$, con este, del conocimiento, pues según ello no podemos obtener conocimiento sino mediante un manejo apropiado de las capacidades lingüísticas. Los límites del lenguaje son los límites del conocimiento. De acuerdo con Lindón \& Hiernaux (2006), “el giro lingüístico replanteaba la concepción de la realidad y del conocimiento". Se abría en las ciencias sociales el camino hacia las visiones constructivistas. Se trata de una perspectiva hermenéutica desde la cual "(...) un texto toma sentido dentro de un contexto"; Velasco, 2012, pp. 202, 203).

También en la epistemología de Piaget-García se plantea una crítica al empirismo científico bajo estos argumentos, según se expone en la siguiente sección de este escrito.

Como resultado de los debates y críticas en torno a la demarcación entre conocimiento científico y otros modos de conocimiento, surgieron distintas propuestas o puntos de vista tendientes a establecer una nueva demarcación. De allí surgen las llamadas metodologías cualitativas en ciencias sociales, orientadas a la comprensión de la vida interior, simbólica o subjetiva de las personas (Bourdieu, 2007; Giddens, 2006; De la Garza \& Leyva, 2012. Dicha vida interior puede caracterizarse como una dimensión simbólica, donde la atribución de significado tanto a las acciones e interacciones sociales como al espacio urbano o arquitectónico, se ha vuelto un tema de conocimiento científico, con lo que podemos referirnos a una reivindicación del sujeto (De la Garza, 2012a, p.230) reflejada, por ejemplo, en las propuestas de la Escuela de Frankfurt, que abordó los problemas de la epistemología “de la forma más sistemática y con mejor conocimiento de las corrientes clásicas y actuales en ese momento en la filosofía y las teorías sociales" (De la Garza, 2012b, p.230). 
De manera paralela, los estudios iniciados por Piaget en la primera mitad del siglo anterior acerca de las teorías del desarrollo, contribuyeron a perfilar una gran corriente de pensamiento en distintas disciplinas que debían responder por las acciones, interacciones o intenciones de los grupos humanos, sociales, culturales, etc. Corriente que ahora reconocemos ampliamente como constructivismo, que se fundamenta, entre otras cosas, en fuentes como Kuhn, Bertalanffy, Morin y, en particular, en la propuesta de Piaget sobre una epistemología genética o constructivista.

Así, con el mismo propósito de conocer el mundo interior de las personas, surgieron nuevos campos de investigación disciplinar, caracterizados por una integración metodológica en respuesta a una realidad cada vez más cambiante y compleja, por ejemplo, las ciencias cognitivas.

Nuestra atención se enfocará ahora en algunas ideas y conceptos clave de Piaget. Según este célebre investigador, el desarrollo de conceptos en los niños durante el aprendizaje continúa en la vida adulta, en un proceso análogo al desarrollo de conceptos en las teorías científicas.

Ello marcó una nueva comprensión del objeto o sentido de la investigación científica, que se resume en la tabla de abajo sobre los modelos del conocimiento, incluyendo la necesidad de comprender para ello los procesos sociales y simbólico-culturales que determinan el comportamiento de las personas, y por ende el contexto donde educamos y formamos a los futuros dirigentes de nuestro mundo.
Como se observará, existen diversas propuestas para abordar estos retos, como las que se plantean en Rapoport (2008), Gallagher (2014), Giddens (2006) o Noë (2010). Y puede decirse que su comprensión y resolución son necesarias para situarnos correctamente ante las circunstancias de nuestro tiempo en lo que concierne al desarrollo cognitivo en los procesos pedagógicos.

Tabla de modelos de conocimiento

\begin{tabular}{|c|c|c|}
\hline & $\begin{array}{l}\text { Positivismo lógico; } \\
\text { Empirismo Cien- } \\
\text { tífico. Dualismo } \\
\text { cartesiano. Filosofía } \\
\text { analítica. Fisicalismo } \\
\text { (o fisicismo). Reduc- } \\
\text { cionismo. }\end{array}$ & $\begin{array}{l}\text { Epistemología Ge- } \\
\text { nética. Pragmatismo } \\
\text { comprensivo. Monis- } \\
\text { mo de doble aspecto. } \\
\text { Filosofía sintética. } \\
\text { Constructivismo. }\end{array}$ \\
\hline Conocimiento & $\begin{array}{l}\text { El lenguaje como es- } \\
\text { pejo de la naturaleza. } \\
\text { Se halla en libros de } \\
\text { texto. Resultado final } \\
\text { planteado en estruc- } \\
\text { turas proposicionales } \\
\text { (lógica). Conoci- } \\
\text { miento a priori. } \\
\text { Modelo Nomotético } \\
\text { (que busca leyes y } \\
\text { verdades) }\end{array}$ & $\begin{array}{l}\text { Conocimiento es len- } \\
\text { guaje; se construye. } \\
\text { Se enfoca a proble- } \\
\text { mas. Conocimiento a } \\
\text { posteriori, enactivo o } \\
\text { experiencial. Modelo } \\
\text { Ideográfico o Com- } \\
\text { prensivo (Interpre- } \\
\text { tativo). }\end{array}$ \\
\hline $\begin{array}{l}\text { Objeto } \\
\text { de estudio }\end{array}$ & $\begin{array}{l}\text { Res extensa. Externo } \\
\text { e independiente al } \\
\text { sujeto. Realidad } \\
\text { física fragmenta- } \\
\text { da y separada del } \\
\text { contexto. Estudio en } \\
\text { laboratorio. }\end{array}$ & $\begin{array}{l}\text { Sistema complejo, en } \\
\text { evolución; el todo es } \\
\text { más que las partes. } \\
\text { Constructo socio- } \\
\text { lingüístico-cultural. }\end{array}$ \\
\hline $\begin{array}{l}\text { Sujeto del } \\
\text { conocimiento }\end{array}$ & $\begin{array}{l}\text { Res cogitans. } \\
\text { Individuo; sujeto } \\
\text { cartesiano. }\end{array}$ & $\begin{array}{l}\text { Colectivo, histórico- } \\
\text { social, interdiscipli- } \\
\text { nar; trans-disciplinar. }\end{array}$ \\
\hline Metodología & $\begin{array}{l}\text { Cuantitativa. Las } \\
\text { experiencias menta- } \\
\text { les vistas desde un } \\
\text { laboratorio, reducidas } \\
\text { a procesos físicos. } \\
\text { Objetivismo. }\end{array}$ & $\begin{array}{l}\text { Cualitativa. Síntesis } \\
\text { entre objetivismo y } \\
\text { subjetivismo. Emo- } \\
\text { ciones, sensaciones, } \\
\text { percepciones y con- } \\
\text { cepciones, en relación } \\
\text { con el contexto. }\end{array}$ \\
\hline
\end{tabular}

El conocimiento desde los puntos de vista del dualismo cartesiano y de una epistemología constructivista. Resumen del autor. Véase, en particular, Bertalanffy (2009), Bourdieu (2007), De la Garza \& Leyva (2012), García (2006), Giddens (2006). 


\section{La discusión sobre la mente y el conoci- miento}

Uno de los retos planteados por la teoría del desarrollo de Piaget se refiere a la caracterización de los recursos cognitivos que hacen posible el aprendizaje, tales como la elaboración de categorías y conceptos (Carey, Zaitchik \& Bascandziev, 2015). En efecto, la conceptualización y la categorización son capacidades que permiten a los seres humanos comprender y organizar el mundo que los rodea, incluyendo sus propias acciones, así como las interacciones simbólicas o comunicativas con otras personas.

En tal situación, tanto el mundo que se nos presenta a los sentidos de la percepción como las demás personas con quienes interactuamos, representan distintos tipos de problemas con los que debemos lidiar. Por ejemplo, entender su discurso, el significado de sus acciones y sus gestos, o de la postura de su cuerpo, y también entender los códigos de una sociedad, su sistema de reglas, los esquemas de comportamiento correctos, las normas sociales y culturales a través del tiempo, etc.

Según González (2006), la facultad o capacidad de simbolización y de lenguaje son evidencia “(...) de una clara capacidad de conceptualización, pues poseer un concepto tiene que ver, por un lado, con una habilidad, específicamente, con una capacidad de reconocimiento" (p.28) del mundo circundante y, por otro lado, con las cualidades o características de las cosas o las personas, en tanto miembros o elementos de grupos.
Pero se puede cuestionar: ¿cómo podemos caracterizar nuestro objeto de estudio, a saber, la facultad de aprender a manejar y elaborar conceptos y categorías, tanto en el conocimiento científico como en el conocimiento ordinario? Y ¿qué es y cómo se genera el cambio conceptual?

Para responder cabalmente a lo anterior, habría que enfocar algunos problemas que parecen aún lejanos de resolver, tales como la relación entre las experiencias perceptuales (percibir el mundo circundante o exterior a las personas), y la facultad de ordenar, representar, categorizar y conceptualizar dicho mundo. Aquí el problema se refiere al aspecto simbólico o significante de tales representaciones, categorizaciones y conceptualizaciones, ya que en particular la dimensión simbólica de las acciones humanas ha propiciado un largo debate respecto de la subjetividad y el comportamiento de las personas, que hoy constituyen un tema de estudio de la epistemología (De la Garza, 2012a).

En este orden de ideas cabe preguntarse: ¿es nuestra mente tan solo el resultado del funcionamiento de un conglomerado de neuronas? Este es el punto de partida de una epistemología empirista, para cuyo objeto de estudio debe reducirse a propiedades físicas y a hechos observables. Considerar el desarrollo cognitivo como la acumulación de competencias individuales en las cuales el orden sociocultural nada tiene que ver, es una idea que ha permeado tanto en las ciencias cognitivas (en su fase inicial) como en la filosofía de las ciencias y la epistemología, 
sobre todo desde el punto de vista del dualismo cartesiano, en el que se fundamenta a su vez el método científico. De acuerdo con esta misma idea, el conocimiento es algo que se busca y surge como resultado final de la investigación, pues, según dicho criterio el conocimiento debe ser "explícitamente formulado en teorías o plasmado en estructuras proposicionales", lo que parece "demasiado estrecho para dar cuenta de la manera real en que el conocimiento crece y se desarrolla" (Eraña, 2009, p.11).

Pero en realidad el crecimiento y desarrollo del conocimiento atañe a toda disciplina científica y humanística, puesto que estas deben usar un bagaje conceptual previamente establecido, que podemos definir como un paradigma, según lo definió Kuhn (2004).

Por lo anterior, si se pretende comprender mejor el desarrollo cognitivo y de las teorías científicas, es fundamental entender lo que en ámbitos de las ciencias cognitivas se llama cambio conceptual y cambio de teorías.

Así, un reto que debe asumir cualquier posición teórica o punto de vista sobre el desarrollo cognitivo consiste en explicar o caracterizar los recursos cognitivos que permiten el aprendizaje, incluyendo la conceptualización y la categorización como capacidades que continúan en la vida adulta, y que también son recursos cognitivos válidos en el conocimiento científico, y aquí, como ya vimos, es donde cobra relevancia el papel de Piaget. Él pensaba que los cambios en la arquitectura cognitiva de los niños durante el desarrollo se constreñía a cambios en el contenido conceptual en distintas edades. En una perspectiva similar, Carey, Zaitchik y Bascandziev (2015, p.4) consideran que el desarrollo de la capacidad conceptual incluye episodios de cambio durante los cuales nuevos recursos representacionales son construidos, lo que a su vez permite pensar en cosas que no se habían pensado antes.

De este modo, parece necesario hallar una respuesta acerca de cómo tiene lugar el cambio de teorías y de conceptos, tanto en los individuos, durante el desarrollo cognitivo y en la vida adulta, como en el desarrollo de teorías científicas. Sin embargo, esa respuesta se relacionará con los enfoques o puntos de vista desde los cuales se asuma lo anterior, por lo que es necesario una caracterización de las principales diferencias entre el dualismo cartesiano y la epistemología constructivista, como se indica en la tabla mostrada anteriormente.

En esa tabla se ofrecen posibles respuestas a las cuales habrá que dar seguimiento, habida cuenta de que por ahora solo podemos plantear los aspectos más relevantes de tan compleja situación.

Terminaremos nuestro escrito con algunos señalamientos provenientes de líneas constructivistas como las de Kuhn, García, Varela y Maturana, quienes, además de Piaget y Morin, entre muchas otras colaboraciones, fundamentan 
las nuevas concepciones o paradigmas sobre el conocimiento y la cognición humana. Un aspecto puntual en este debate se refiere al papel que juega el contexto de los sujetos en la adquisición del conocimiento, que podemos resumir en la frase: conocer es participar (Di Paolo, 2013).

\section{La integración disciplinar constructivista}

Se han presentado someramente algunas diferencias de fondo entre dos maneras de entender el conocimiento, poniendo atención a las analogías entre el conocimiento científico y el conocimiento ordinario en cuanto a los procesos de cambio de teoría y cambio conceptual. Como señalamos antes, desde el punto de vista del positivismo lógico y de la filosofía analítica anglosajona, el contexto de las personas no juega ningún papel en el desarrollo de conceptos y teorías, pero hay cada vez más opiniones favorables acerca de lo contrario, mismas que revisaremos enseguida.

Todo apunta hacia la integración disciplinar mencionada también con relación a la obra de Piaget y del desarrollo de las ciencias cognitivas, pero examinando tal desarrollo histórico podemos tener más claridad en cuanto a las divergencias en los modelos del conocimiento (García, 2006, p.77). En este sentido, una manera de comprender mejor las diferencias entre los paradigmas cartesiano y constructivista acerca del cambio conceptual y de teorías es la ponderación del papel que juega el contexto en que se desenvuelven los sujetos: el mundo de vida tal como cada persona lo experimenta y le da sentido. De hecho, este punto de vista subjetivo no fue tomado en cuenta en las discusiones epistemológicas sino hasta la segunda mitad del siglo anterior, como se ha señalado anteriormente. Dado, además, que las llamadas metodologías cualitativas en ciencias sociales se interesan por cuestiones como la cultura, los significados o la capacidad de representación y simbolización, ello puede ayudar a comprender el papel que juega el contexto sociocultural en los procedimientos heurísticos, ya sea de la investigación científica, como en el desarrollo cognitivo de los niños, y en el conocimiento ordinario o no científico.

Así, durante la segunda mitad del siglo anterior y gracias al desarrollo de metodologías cualitativas en ciencias sociales, se encontró que el modelo individualista del desarrollo cognitivo como acumulación de competencias individuales en las cuales el orden sociocultural no tenía valor alguno, no era suficiente para explicar la transmisión del conocimiento.

Autores como Feyerabend (2008) o Kuhn (2004) planteaban al respecto que la epistemología de la ciencia debía tomar en cuenta aspectos relacionados con la historia de las disciplinas y con la interacción de los científicos entre sí y con otras comunidades de investigadores, así como el impacto del acceso masivo a la información en la producción del conocimiento, o el papel de la autoridad en los procesos de justificación de las creencias (Eraña, 2009, p.12).

No en balde Kuhn (2004, p.293) había 
llamado la atención sobre cómo la noción de paradigma permite aglutinar los esfuerzos de la comunidad científica, la cual es al mismo tiempo un grupo de personas con sus propias características subjetivas. Esta reflexión le llevó, junto a diversos filósofos e investigadores de incipientes fusiones disciplinares, a pensar en el conocimiento como una construcción social.

Así, junto a investigadores de la mente, la psicología y la cognición, como Alexander Luria, Lev Vygotsky, Chomsky (citados en Carey, Zaitchik \& Bascandziev, 2015), también Piaget habían examinado los diversos procesos y estadios del desarrollo cognitivo, por ejemplo, la facultad de usar el lenguaje y de conceptualizar y categorizar por parte de los niños, y entre otros importantes aportes al conocimiento universal, el constructivismo de Piaget permitió comprender con mayor amplitud la capacidad de los seres humanos para elaborar conceptos con lo que se entendió mejor el desarrollo de nuestras propias teorías para descifrar los enigmas que se presentan ante el conocimiento.

Recordemos que los conceptos nos ayudan a reconocer el mundo, y la conceptualización es por ello una capacidad de reconocimiento (González, 2006, p.28). De esta forma, el problema de la conceptualización apenas se hace visible cuando queremos esbozar una hipótesis para tratar de explicar algún aspecto de la realidad, por ejemplo, al reconocer algún tipo de sustancia.

En otro sentido, las ciencias sociales se enfrentan a la cuestión de las interacciones y las representaciones simbólicas y su estatuto epistémico, un tema debatido largamente que intersecta diversas disciplinas interesadas en responder por la subjetividad de los actores sociales y que también ha propiciado un rebasamiento conceptual del paradigma cartesiano, el empirismo científico y la filosofía analítica. Se debe señalar, así, que tanto las interacciones comunicativas como las representaciones simbólicas implican un procedimiento de interpretación que requiere de conceptos y teorías en la forma de conocimiento no ordinario, y con heurísticas propias. Bourdieu (2007) lo hace, por ejemplo, mediante conceptos como sentido práctico y habitus, Giddens (2006) con su propuesta de análisis de la acción social y de los esquemas cognitivos, y así otros ejemplos en ciencias sociales.

Por otra parte, los investigadores sociales no tienen otra opción sino interpretar lo que hacen y dicen las personas. Esto hace que “(...) los conceptos empleados por los científicos sociales se encuentren enlazados en forma indisoluble con la comprensión que tienen los actores sociales" (De la Garza \& Leyva, 2012, p.30).

Algunos filósofos han planteado que las cuestiones acerca de los conceptos, ya sea que se trate de conceptos constitutivos de cierto paradigma del conocimiento o de cierta estructura conceptual para caracterizar fenómenos sociales, naturales o simbólicos pertenecen al ámbito de la filosofía y no al de la ciencia. En particular 
cuando debemos examinar, como sucede hoy, una realidad compleja, las relaciones estructurales entre distintos campos conceptuales, por ejemplo entre psicología, cerebro, comportamiento y mente, estamos en terrenos de la filosofía (Bennett \& Hacker, 2007, p.4). Aún más, las cuestiones conceptuales conciernen más bien a formas o maneras de representación que a cuestiones de verdad o falsedad, lo cual es de mayor trascendencia cuando se pretende adscribir atributos psicológicos al cerebro (Bennett \& Hacker, 2007 p.6).

Otro problema a considerar es el de las categorías conceptuales y su propia naturaleza, para lo cual se cuenta hoy día con el aparato teórico que llamamos ciencias cognitivas, una conjunción disciplinar donde podemos apreciar con más detalle las diferencias entre los paradigmas cartesiano y constructivista.

Según Lakoff (2012), mientras que la ciencia cognitiva de primera generación llevaba a cabo el programa filosófico del empirismo cartesiano, la de segunda generación no presupone una filosofía a priori, sino "tomarse seriamente la investigación empírica, demandar las generalizaciones más amplias y buscar pruebas convergentes de muchas fuentes" (p.20).

Y es que, como apunta García (2006), no es lo mismo una ciencia empírica que un empirismo científico, pues, un punto de vista empirista afirma que la investigación científica se basa en hechos observables, mientras la ciencia empírica, desde el constructivismo, plantea en primer lugar una crítica conceptual para determinar que no hay hechos observables sino solo interpretaciones por parte de los investigadores.

Hablando de los procesos cognitivos y mentales de los individuos, el llamado "supuesto cartesiano" se refiere a pensar o caracterizar la cognición, tanto en las ciencias empíricas como en la filosofía, bajo un dualismo en el que, por un lado, se tiene "un agente individual, provisto de una mente activa (...) y por otro un entorno pasivo en el que la mente busca información que (a través de una interfase representacional) constituye conocimiento pertinente para la toma de decisiones" (Martínez, 2009, pp.21, 22).

Tomemos el caso que refiere García (2006, p.21) de cómo el racionalismo cartesiano que fundamentaba el sistema educativo francés fue cuestionado severamente por las propuestas de Edgar Morin. En efecto, para Morin el paradigma cartesiano basado en la disyunción entre ego cogitans (el sujeto pensante) y res extensa (la cosa sobre la cual se piensa), mismo que "controla la aventura del pensamiento occidental desde el siglo XVII, ha permitido, sin duda, los enormes progresos del conocimiento científico y de la reflexión filosófica" (Morin, 2007, pp.29 y 30), lo que habría llevado a la ciencia a un desconocimiento de sí misma, y a aislar radicalmente "los tres grandes cambios del conocimiento científico: la física, la biología, la ciencia del hombre" (Morin, 2007, p.29).

Pero para García ello no satisface la 
necesidad de formular de manera precisa ciertos problemas que "el cartesianismo dejó pendientes y que corresponden al campo de la teoría del conocimiento", como son aquellos aspectos metodológicos conducentes o apropiados en las situaciones específicas que Morin considera complejas (García, 2006), y que aquí podemos asociar con las heurísticas constructivistas que referiremos en los resultados del presente escrito.

Como sigue planteando García (2006), para el empirismo científico la búsqueda de leyes, teorías y generalizaciones tenía sentido en la medida en que se hiciese referencia a un mundo observable, a un mundo de hechos físicos. Esta herencia de Francis Bacon dominó la filosofía de la ciencia durante la primera mitad del siglo XX y aún hoy, después de los cambios ocurridos desde la mitad del siglo anterior, persiste en "el pensamiento de los científicos no habituados a la reflexión epistemológica" (p.40).

El cambio conceptual requiere pues ser examinado a la luz de una perspectiva distinta a la del cartesianismo, es decir, desde una visión que cuestione el modelo individualista de la cognición y el conocimiento, para afirmar el importante papel que el contexto social juega en la generación y cambio de conceptos y teorías.

Así, con Kuhn se consideran como aspectos propios del conocimiento científico "no solo la construcción de artefactos, teorías o textos científicos", sino también la construcción social del mundo a que se refieren las teorías científicas, y con el que interactúan los científicos (León Olivé, citado por Retamozo, 2012, p.377). Y por ello nos referimos antes a los procedimientos heurísticos tanto en el sentido de búsqueda del conocimiento como en el de los recursos materiales y metodológicos conducentes.

Como ejemplo existen diversas propuestas en ciencias cognitivas, en ciencias sociales, y en ciencias ambientales y del comportamiento, que comparten este punto de vista. Es posible citar entre ellas la noción de consiliencia de Edward O. Wilson y Amos Rapoport (Rapoport, 2008), la racionalidad ecológica del filósofo Gerd Gigerenzer y el grupo ABC (Martínez, 2009, p. 21), la llamada cognición situada (Dokic, 2006), la mente corporizada (Lakoff, 2012), el enactivismo y la autopoiesis (Maturana \& Varela, 2003; Di Paolo, 2013), la sociología fenomenológica de Shaun Gallagher (2014) o las llamadas geografías constructivistas, de cuño reciente (De la Garza \& Leyva, 2012).

Otro ejemplo es el de una epistemología social, aquella encargada de examinar no solo el papel de los sujetos o actores sociales en el conocimiento, sino la cuestión de si los contenidos del conocimiento se encuentran "en la cabeza" de las personas o distribuido entre ellas (Eraña, 2009 p.17).

Esta última problemática no es fácil de resolver, ya que no se ha encontrado una correlación entre los procesos bioquímicos del cerebro y lo que arriba mencionamos como una interfase representacional. Es decir, aun cuando está en boga la concepción constructivista, tanto acerca del conocimiento como de la 
sociedad y el mundo subjetivo de las personas, no se sabe qué eventos específicos del cerebro corresponden a los eventos mentales de las experiencias individuales. Ciertamente lo que Di Paolo llama interfase representacional dista de ser comprendida, toda vez que hay opiniones encontradas acerca del carácter epistémico de las representaciones simbólicas, de los objetos simbólicos, de los significados y de la facultad de asignar significado al mundo circundante y a las acciones de los demás.

Para todo ello, se ha recurrido a fenomenólogos como Maurice Merleau-Ponty para determinar el papel de la corporalidad, esto es, de la capacidad perceptual del cuerpo humano, en el conocimiento. Es conocido el ejemplo de este filósofo cuando se refirió al fútbol para plantear su concepción sobre la influencia del cuerpo en las heurísticas del conocimiento.

Siguiendo con la metáfora de MerleauPonty, "las intenciones y las acciones del jugador son moldeadas por el entorno físico y por la naturaleza del juego que está haciendo" (Gallagher, 2014, p.237).

A diferencia de Francis Crick, para quien "nuestro sentido de identidad personal no es sino resultado del comportamiento de un amasijo de células nerviosas", el neuro-filósofo Alva Noë (2010, p.21) piensa, como MerleauPonty y muchos otros, que la conciencia no solo está dentro sino también fuera de la cabeza. Es un error buscar correlatos neuronales a la conciencia, dice Noë, pensando que se trata de "estructuras o de procesos neuronales que son suficientes por sí solos para generar consciencia” (p.223).

Del mismo modo, en ciencias cognitivas se echa mano de conceptos clave que ya se habían desarrollado en la fenomenología, en la hermenéutica, semiótica, ontología, tales como el de mundo de vida, usado para referirse al espaciotiempo donde suceden las experiencias subjetivas de las personas.

Una reelaboración o elucidación conceptual para relacionar distintas áreas disciplinares interesadas en este tema es, por ejemplo, el caso de la sociología fenomenológica, que aquí se ejemplifica con Gallagher. La cognición social, en efecto, se refiere al conjunto de capacidades que nos permiten entender las intenciones de otros y el significado de sus acciones (Gallagher, 2014, p.231). Pero la cognición social es solo un aspecto de la atención conjunta, la cual involucra también aspectos emocionales orientados a la acción, y se refiere a una relación intersubjetiva donde se aplican capacidades sensoriomotoras para comprender el significado de los movimientos, gestos, dirección de la mirada y otras acciones intencionales de las personas en su relación cotidiana.

Desde este punto de vista, la atención conjunta tiene que ver con la forma como se nos presenta a los sentidos el mundo que nos rodea, una situación que tiene lugar durante el desarrollo 
cognitivo hacia los nueve meses de edad (Gallagher, 2014, p.226), y que continúa durante la vida adulta. Como vemos, las facultades cognitivas de conceptualizar son un aspecto de una totalidad compleja donde interviene también el lenguaje y lo social, la percepción y la categorización, la memoria e, incluso, las emociones.

El enactivismo, otra perspectiva crítica al empirismo científico cartesiano, plantea este mismo punto de vista, al considerar a la cognición como una actividad continua moldeada por procesos auto-organizados de participación activa en el mundo y por la experiencia y autoafección del cuerpo animado (Di Paolo, 2013).

Para Lakoff (2012, pp.19, 33) la mente está encarnada en el sentido de que "la estructura de los conceptos y los mecanismos de la razón surgen, en última instancia, del sistema sensoriomotor del cerebro y el cuerpo, que les dan forma". Lo que aquí nos interesa señalar es que, a diferencia de los modelos del conocimiento que continúan con la herencia de Bacon y del cartesianismo positivista, el constructivismo, en las distintas maneras que mencionamos, "recupera y replantea problemas que son clásicos en la reflexión sobre los modos en que se produce el conocimiento, tanto en términos generales (gnoseología), como por la actividad científica" (Retamozo, 2012, p.374).

En tal sentido, una situación en que divergen la ciencia empirista y la epistemología constructivista es en la concepción del objeto de estudio, ya que en la medida que dicha concepción es precisamente una construcción conceptual y teórica, todo parte del uso o interpretación que se hace de tales conceptos y teorías.

Mediante sólidas investigaciones epistemológicas propuestas desde la psicología genética, Piaget había demostrado que no hay observables puros, es decir, que todo observable, aun aquellos que parecen provenir de la percepción directa de las propiedades elementales de los objetos, suponen una previa construcción de relaciones por parte del sujeto (García, 2006, pp.41, 42).

Según De la Garza y Leyva, (2012, p.20), "incluso con el auxilio de la lógica y de las matemáticas, hubo que reconocer finalmente que la ciencia maneja dos tipos de lenguaje: el teórico y el observacional", por lo cual no siempre podemos hablar de conceptos científicos directamente observables.

El argumento de Piaget-García es que no podemos conocer la realidad tal como es en sí misma mediante nuestros sentidos, sino a través de construcciones conceptuales culturalmente aprendidas. Cuando se elige un aspecto de la realidad para ser examinado, y más en las realidades complejas contemporáneas, no se puede hablar de hechos directamente observables, pues en ese caso todos sacarían las mismas conclusiones de sus estudios, pero, por el contrario, ya se ha visto cómo difieren los puntos de vista sobre el conocimiento mismo.

Según esto, no hay una propuesta metodo- 
lógica en la información anterior, pero existen obvias implicaciones metodológicas. Afirma García (2006, p.42) que pueden ser definidas como antiempiristas en tanto se niega que las características del sistema estén dadas y sean accesibles a la experiencia directa de cualquier "observador neutro".

De este modo, un enfoque interdisciplinar plantea algunas diferencias con Morin y Wallerstein, otros pioneros en los estudios de la complejidad en ciencias sociales, pues, en primera instancia, una sola investigación disciplinar por sí misma no puede asumir el punto de vista de otras disciplinas.

No basta que los conocimientos de dominios distintos de la realidad se acerquen o se pongan juntos, dice García, sino que más bien "los procesos de integración disciplinaria (al igual que los procesos de diferenciación que han dado lugar a cada una de las disciplinas científicas), han significado replanteamientos fundamentales" (García, 2013, p.201). Así pues, tenemos distintos puntos de vista acerca del cambio conceptual, mismo que planteamos al principio como uno de los recursos cognitivos en el desarrollo de teorías y heurísticas para la obtención de conocimiento, tanto si hablamos de un conocimiento del sentido común como del científico, pues podemos confirmar que hay una continuidad entre ambos (Eraña, 2009, p.15).

Según Susan Carey (como se cita en Eraña, 2009, pp.63 y 65), uno de los puntos de vista acerca de qué es y cómo se genera un cambio conceptual distingue entre conocimiento central y conocimiento construido, y plantea la existencia de dos tipos de estructuras o mecanismos cognitivos: por un lado, los módulos centrales cuyo desarrollo implica un enriquecimiento de la base conceptual inicial; por otro lado, las teorías intuitivas, que resultan de un cambio conceptual, como es el caso del constructivismo.

Deesta manera, mientras los módulos centrales permanecen estables durante el desarrollo, y son resultado de la evolución biológica, lo cual es compartido con algunas especies de primates, las teorías intuitivas (las que postulan la importancia del contexto) difieren de la teoría de los módulos centrales en tanto las primeras implican procesos de innovación y "adquisición de nuevas creencias establecidas sobre conceptos nuevos" (Eraña, 2009, p.16).

George Lakoff, pionero de la lingüística cognitiva, examinó detalladamente junto con Mark Johnson los resultados de más de dos mil quinientos años de especulaciones filosóficas "acerca de las vidas cotidianas de las personas ordinarias" (Lakoff, 2012, p.27), para llegar a una mayor comprensión de los estados de conciencia, como los que corresponden a cambios conceptuales y de teorías. Acerca de ello, una de las valiosas aportaciones de Giddens propone que los conceptos que los científicos sociales utilizan para hablar de la realidad social "se encuentran enlazados en forma indisoluble con la comprensión que tienen los actores 
sociales" (De la Garza y Leyva, 2012, p. 30) y que les permite orientarse, interpretar su realidad, y actuar en su vida cotidiana.

Así pues, para hablar del conocimiento debemos incluir también la subjetividad del significado de las acciones sociales y de la cultura como dimensión simbólica, que juega algún papel en lo que podemos llamar la construcción social del conocimiento, si se enfoca desde un punto de vista experiencial, interpretativo y comprensivo. En consecuencia, podríamos afirmar que "la expresión del pensamiento es entonces una entidad 'híbrida', hecha de signos lingüísticos pero también de circunstancias no lingüísticas" (Dokic, 2006, p.113), tales como las acciones e interacciones sociales. Y se trata también, como señalan Maturana y Varela (2003, p.164), no solo del interés por la exploración científica "sino que nos entrega la comprensión de nuestro ser humano en la dinámica social".

Entre otros ejemplos a nivel regional está el de Bacca (2014, p.71), Monterroza, (2014, p.105), Ospina (2016, p.108), Peñaloza (2015, p.314) y Feo (2015, p.221), donde se enfatiza que: el aprendizaje está relacionado con el contexto socio-cultural, el docente aplica su propia experiencia, el aprendizaje continúa en la vida adulta, y el conocimiento se construye socialmente.

Por todo lo anterior, el conocimiento científico sería mejor comprendido en su desarrollo y crecimiento si pudiese entenderse su continuidad con el conocimiento del sentido común, por lo cual propusimos en este escrito abordar dicha cuestión, observando el desarrollo de procedimientos heurísticos basados en elucidaciones conceptuales que redunden en cambios de teorías, y por lo tanto, en una mejor comprensión de la realidad.

\section{Conclusiones}

Sin duda todo cambio conceptual es consustancial a un cambio de teorías y eventualmente a un cambio de paradigma o concepción del conocimiento, pero aún se discute la manera en que puede ocurrir ello, siendo cada vez más aceptado un modelo del conocimiento en términos de una teoría de la evolución biológica, tal como Piaget lo planteó en la epistemología genética. Según esto, el conocimiento es enactivo, depende del contexto socio-cultural y por lo tanto es una construcción lingüística y social (Véase Peñaloza, 2015, p.309; Feo, 2015, p.222)

Así, una primera consecuencia de este rebasamiento conceptual y cambio de teorías, desde un punto de vista cartesiano-empirista a uno constructivista, sugiere que existen aspectos del conocimiento que se alejan de una racionalidad científica, misma que al tener necesidad de trabajar con conceptos y teorías históricamente determinadas, se ve también en la necesidad de revisar la historia de las ideas, y adoptar así una perspectiva crítica y filosófica. Como refirió el reconocido epistemólogo mexicano León Olivé, para el constructivismo "no hay producción de conocimiento por fuera de las teorías, conceptos, y metodologías que utilizamos para la construcción y abordaje de nuestro objeto de estudio" (Retamozo, 2012, p.390). 
Como puede verse, existen varias coincidencias entre la filosofía pragmatista, entre cuyos detentadores principales se hallan Dewey, Peirce, Merleau-Ponty, Heidegger y Wittgenstein, además de las propuestas sobre la cognición corporizada, el enactivismo, las prácticas ecológicas, la cognición situada, y otras corrientes actuales, que no difieren sustancialmente de la epistemología constructivista de Piaget-García, ya que según esta, "conocer significa establecer relaciones en una materia prima que, sin duda, es provista por la experiencia, pero cuya organización depende del sujeto cognoscente" (García, 2006, p.43).

Se confirma también que la conceptualización, como paso previo al desarrollo y cambio de teorías, es sin duda solo un aspecto de un proceso más complejo al que podemos llamar cognición. Dicha cognición comprende también la percepción y la categorización (González, 2006, p.11), cuyo estudio rebasa distintas disciplinas interesadas en las acciones humanas, y en la actualidad permite hablar también de un cambio de paradigma en el conocimiento, al entenderlo como una construcción, al igual que el objeto de estudio y las metodologías para conocerlo.

Por ello, una de las críticas al dualismo cartesiano concierne a sus sistemas conceptuales, que a su vez sustentan los procedimientos heurísticos y metodológicos en la obtención del conocimiento, al concebirlos como un proceso separado de su contexto sociocultural. Por ejemplo, en el caso de la disyunción sujeto-objeto (o mente y materia), y la idea de que existen hechos neutros empíricamente observables. Ello ocurre en campos del saber que pretenden asumir una metodología científica en terrenos donde el objeto de estudio no es físico, por ejemplo, en lo social y lo simbólico.

Por lo tanto parece necesario señalar la importancia de las categorías conceptuales que empleamos, ya sea en el conocimiento ordinario o en el científico, y agregar también que la reflexión sobre los conceptos y teorías plantea distintos retos, uno de ellos es su carácter complejo e interdisciplinar. Este es el caso cuando tenemos por una parte el problema de la existencia de las categorías conceptuales, que al mismo tiempo son categorías ontológicas cuyo estudio evidentemente no corresponde a un enfoque cientificista (De la Garza y Leyva, 2012, p.21; Peñaloza, 2015, p.318), sino humanístico y filosófico. Por otra parte, ante el hecho de que la capacidad cognitiva de categorizar se presenta como una actividad neuronal compleja en interacción con el medio físico, social, y simbólico, debe reconocerse que su estudio sí corresponde al enfoque de las ciencias empíricas, y que ante tal situación es que mencionamos antes el papel de las integraciones disciplinares como son los casos de las ciencias cognitivas, la neurofenomenología, o la fenomenología social.

Si la ciencia maneja dos tipos de lenguaje, teórico y observacional, entonces pierde valor el supuesto de que hay conceptos científicos directamente observables y en cambio se asume que el conocimiento y el lenguaje son interdependientes, pues las categorías conceptuales 
permiten organizar o "acomodar" los objetos del mundo físico y también su conocimiento mismo. Por ello, desde un punto de vista constructivista, "el problema metodológico consiste en cómo descubrir categorías propias de un objeto no universal y que sean capaces de expresar su origen, su funcionamiento y contradicciones", como señaló ya De la Garza (2012a, p.236).

Sin duda esto implica también cierta relativización del conocimiento en cuanto a la pretensión de verdad como cosa independiente del contexto cultural, es decir, en términos de un modelo nomotético que enfatiza la pretensión y consecución de verdades y leyes universales, mediante criterios de las ciencias físicas, en lugar de plantearse como resultado de la experiencia de vida, en el sentido en que distinguimos entre conocimientos a priori y a posteriori.

Vemos pues que, en el caso de objetos de estudio que no son físicos, como lo social y lo simbólico, su caracterización depende de la experiencia del investigador en su propio contexto socio-cultural (del cual aprende esquemas culturales y conceptuales). Por ello puede hablarse de heurísticas que a través de la interacción social y simbólica permiten conocer nuevos aspectos de la realidad circundante, mediante la enacción o el aprender haciendo, participando, interviniendo en el mundo de todos los días. Se trata de un mundo que se aparece, sí, ante nuestros órganos de la percepción, pero que es interpretado mediante construcciones conceptuales culturalmente aprendidas.

\section{Referencias}

Bacca, A. (2014). La educación no formal contextualizada: huellas de su desarrollo y transformación en Cúcuta. Revista Educación y Humanismo, 16(27), 70-86, 71.

Bachelard, G. (2005). El Compromiso Racionalista. Distrito Federal, México: Siglo XXI.

Bennett, M. R. \& Hacker, P.M.S. (2007). Philosophical Foundations of Neuroscience. En: M. Bennett, et al. Neuroscience \& Philosophy. Brain, Mind, and Language. New York, USA: Colombia University Press.

Bertalanffy, L. (2009). Teoría General de los Sistemas. Distrito Federal, México: Fondo de Cultura Económica.

Bourdieu, P. (2007). El sentido práctico. Buenos Aires, Argentina: Siglo XXI.

Carey, S., Zaitchik, D. \& Bascandziev, I. (2015). Theories of Development: In Dialog with Jean Piaget. Developmental Review, 38, December, 36-54. Recuperado de: http:// fulltext.study/article/353441/Theories-ofdevelopment-In-dialog-with-Jean-Piaget el día 23 de enero de 2017. Véase también: https://www.researchgate.net/publication/282464640_Theories_of_development_In_dialog_with_Jean_Piaget, recuperado el día 25/10/16.

De la Garza, E. (2012a). La Metodología Marxista y el Configuracionismo Latinoamericano. En: E., De la Garza, y G. Leyva, (editores), Tratado de Metodología de las Ciencias Sociales (pp.229255). Distrito Federal, México: Universidad Autónoma Metropolitana-Fondo de Cultura Económica.

De la Garza, E. (2012b). Grounded Theory. 
Cantidad, calidad, y comprensión de significado. En: E., De la Garza, y G., Leyva, (pp.397-419). Tratado de metodología de las Ciencias Sociales Distrito Federal, México: Universidad Autónoma Metropolitana Fondo de Cultura Económica.

De la Garza E. \& Leiva, G. (2012). Tratado de metodología de las Ciencias Sociales, Distrito Federal, México: Universidad Autónoma Metropolitana Fondo de Cultura Económica.

Di Paolo, E. (2013). El enactivismo y la naturaleza de la mente. En D. P. Chico y M. G. Bedia (coords.). Nueva ciencia cognitiva: Hacia una teoría integral de la mente. Madrid, España: Plaza y Valdés. Recuperado de https:/ezequieldipaolo.files.wordpress. com/2011/10/enactivismo_e2.pdf, el día 27/11/16.

Dokic, J. (2006). Representaciones situadas de objetos y del espacio. En C., J. González (editor). Perspectivas contemporáneas de la cognición. Distrito Federal, México: Siglo XXI.

Eraña, Á. \& Mateos, G. (2009). Dos variedades de contextualismo. En Á., Eraña, G. Mateos, La Cognición como Proceso Cultural. (pp.99-123). Distrito Federal, México: Universidad Nacional Autónoma de México.

Eraña, A. (2009). Dos Explicaciones Alternativas del Cambio Conceptual. En A., Eraña, y G. Mateos, (coords.). La Cognición como Proceso Cultural. (pp.63-89). Distrito Federal, México: Universidad Autónoma de México.

Feo, R. (2015). Epistemología y práctica de la investigación sobre el aprendizaje estratégico en América Latina. Revista Educación y Humanismo, 17(29), 220-235. http://dx.doi.org/10.17081/ eduhum.17.29.1254

Feyerabend, P. (2008). La Ciencia en una Sociedad Libre. Distrito Federal, México: Siglo XXI.

Fuentes, F. (2011). La Experiencia Cualitativa en el Paisaje y el Patrimonio Construido. Apuntes. Revista de Estudios sobre Patrimonio Cultural, 24(2), 168-177. Véase: www.javeriana.edu.co/apuntes

Fuentes, F. (2013). Aspectos Intangibles del $P$ 'urhé echerio. Hacia un estudio integral de los paisajes de la Cuenca Lacustre de Pátzcuaro. Morelia, México: Universidad Michoacana de San Nicolás de Hidalgo.

Fuentes, F. (2015). Constructos y Paisajes de una Ciudad Histórica. Revista Contexto. Universidad Autónoma de Nuevo León. IX (10), marzo, 57-68.

Fuentes, F. (2015). Entre el Espacio Público y el Paisaje Urbano. Revista Arquitectura y Cultura, (7), 47-59. Universidad Santiago de Chile.

Gallagher, S. (2014). Coordinación y creación de sentido en la atención conjunta y la acción conjunta. En P., King, J.,González, y E. González, (coords.) Ciencias cognitivas y filosofia. Entre la cooperación y la integración. Distrito Federal, México: UAQ-Porrúa.

García, R. (2006). Sistemas Complejos. Barcelona, España: Gedisa.

García, R. (2013). Investigación interdisciplinaria de sistemas complejos: lecciones del 
cambio climático. Voces Cardinales, 1(1), 193-206.

Giddens, A. (2006). La constitución de la sociedad. Buenos Aires, Argentina: Amorrurtu.

González, J. (2006). Presentación: "La cognición como objeto de estudio filosófico y científico". En: C., J. González, (editor). Perspectivas contemporáneas de la cognición. Distrito Federal, México: Siglo XXI. (pp.11-36).

Kuhn, T. (2004). La Estructura de las Revoluciones Cientificas. Distrito Federal, México: Fondo de Cultura Económica.

Lakoff, G. (2012). Filosofía de carne y hueso. En J. Brockmann, (editor). Mente. (pp.13-33) Barcelona, España: Crítica.

Lindón, A. \& Hiernaux, D. (2006) Tratado de geografía humana, Anthropos. México: Universidad Autónoma MetropolitanaUnidad Iztapalapa.

Martínez, F., S. (2009). Hacia una racionalidad ecológica distribuida en prácticas. En: A., Eraña, y G. Mateos (pp.21-53). La cognición como proceso cultural: Distrito Federal, México: Universidad Nacional Autónoma de México.

Maturana, H. \& Varela, F. (2003). El Árbol del Conocimiento. Las Bases Biológicas del Entendimiento Humano. Buenos Aires, Argentina: Lumen-Editorial Universitaria. Monterroza V. (2014). Modelo pedagógico social cognitivo y su aplicación en las prácticas pedagógicas de docentes y estudiantes del programa de formación complementaria de la Institución Educativa Normal Superior de Sincelejo. Revista Educación y Humanismo, 16(26), 104-121.
Morin, E., (2007). Introducción al Pensamiento Complejo. Barcelona, España: Gedisa.

Noë, A. (2010). Fuera de la cabeza. Porqué no somos el cerebro y otras lecciones de la biología de la consciencia. Barcelona, España: Kairós.

Ospina, V. (2016). El docente del nivel inicial: retos para la formación profesional y continua. Revista Educación y Humanismo, 18(30), 107-122. http://dx.doi.org/10.17081/ eduhum.18.30.1325 p. 108

Peñaloza, G. (2015). Una mirada desde la Didáctica de las Ciencias al concepto de visión del mundo. Revista Educación y Humanismo, 17(29), 308-320. http:// dx.doi.org/10.17081/eduhum.17.2

Rapoport, A. (2008). Perspectivas y Referencias. Las relaciones entre la mente, el territorio y la sociedad desde una perspectiva medioambiental. En: J. Muntañola, Mente, Territorio y Sociedad. (pp.33-62) Barcelona, España: Universidad Politécnica de Cataluña.

Retamozo, M. (2012). Constructivismo: epistemología y metodología de las ciencias sociales. En: E., De la Garza y G. Leyva, (eds.). Tratado de la metodología de las Ciencias Sociales (pp. 373-395). Distrito Federal México: Universidad Autónoma Metropolitana Fondo de Cultura Económica

Velasco, A. (2012). Hermenéutica y Ciencias Sociales. En: E., De la Garza, y G. Leyva, (eds.). Tratado de metodología de las ciencias Sociales (pp.199-228) Distrito Federal México: Universidad Autónoma Metropolitana Fondo de Cultura Económica. 